\title{
Piezotronic Effect on ZnO Nanowire Film Based Temperature Sensor
}

\author{
Fei Xue, ${ }^{\dagger}$, Limin Zhang, $^{\dagger, \S}$ Wei Tang, ${ }^{\dagger}$ Chi Zhang, ${ }^{\dagger}$ Weiming Du, ${ }^{\dagger}$ and Zhong Lin Wang ${ }^{*}, \dagger, \ddagger$ \\ ${ }^{\dagger}$ Beijing Institute of Nanoenergy and Nanosystems, Chinese Academy of Sciences, Beijing, 100083, China \\ ${ }^{\ddagger}$ School of Material Science and Engineering, Georgia Institute of Technology, Atlanta, Georgia 30332, United States
}

Supporting Information

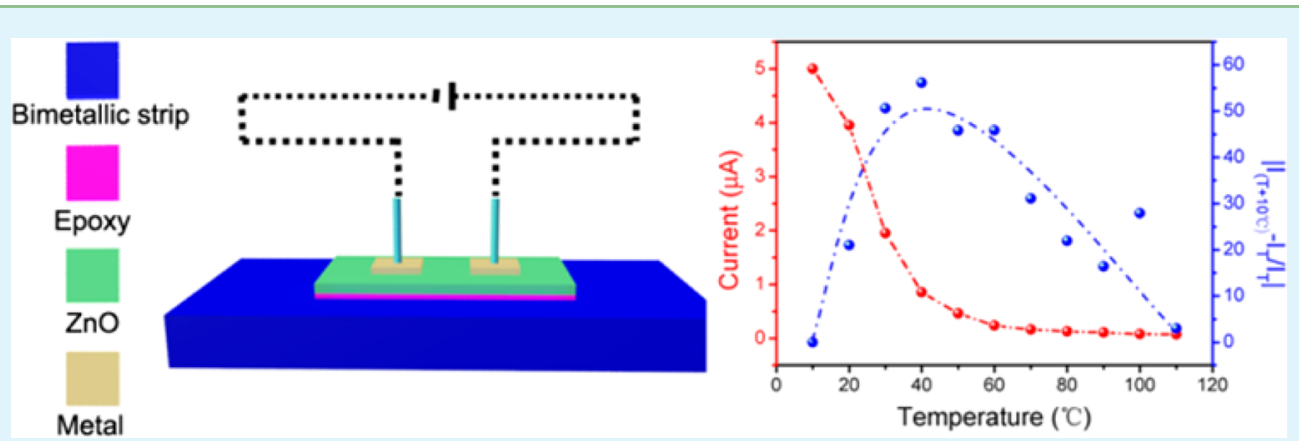

ABSTRACT: In this work, we demonstrated the first study of piezotronic effect as a potential means for measuring temperature by utilizing $\mathrm{ZnO}$ nanowire (NW) film. The film was synthesized by the wet chemical deposition method and transferred to a flexible substrate using photoresist. The primary role of piezotronic effect over geometrical and piezoresistive effect in the asfabricated devices has been confirmed, and piezotronic effect on charge carrier transportation under different strains is subsequently studied. In addition, we also presented that the temperature sensing capability of as-fabricated NW film based piezotronic devices can be tuned by piezopotential, which exhibits dramatically enhanced sensitivity. A theoretical model is proposed to interpret the observed behaviors of the sensor. This study provides an effective method to fabricate temperature sensors with higher performance based on piezotronic effect in the future.

KEYWORDS: piezotronic effect, $\mathrm{ZnO}$ nanowire film, wet chemical deposition, temperature sensor, bimetallic strip

\section{INTRODUCTION}

Recently, Wurtzite-structured piezoelectric semiconductors, such as $\mathrm{ZnO}, \mathrm{GaN}$, and $\mathrm{InN}$, have been of particular interest owing to their unique potential in novel applications by conjunction of piezoelectric effect and semiconductor properties. $^{1-3}$ Due to the noncentral symmetric hexagonal structure, a Wurtzite-structured material generates a piezoelectric potential across the crystal under strain. Piezoelectric-polarizationinduced piezopotential in strained piezoelectric semiconductors acts as a "gate" to modulate carrier transportation across the metal-semiconductor interface or $\mathrm{p}-\mathrm{n}$ junction. This modulation effect has produced a new mechanism for influencing the charge carrier transportation process, in analogy to the wellknown externally electrically induced "field-effect transistor," which was first proposed as the piezotronic effect in $2007 .{ }^{4} \mathrm{~A}$ series of piezotronic effect-based devices have been fabricated, including strain sensors, ${ }^{5}$ piezopotential gated transistors, ${ }^{6,7} \mathrm{PH}$ sensors, ${ }^{8}$ gas sensors, ${ }^{9}$ biosensors, ${ }^{10}$ and so forth, which exhibits great potential in applications.

As of now, most of the fabricated piezotronic devices are based on one-dimensional (1D) Wurtzite nanostructures, especially $\mathrm{ZnO}$ nanowires. Although $1 \mathrm{D}$ nanostructures are attractive to fundamental studies and possibly future electronics, they possess certain limitations for industrial applications. So far, various techniques have been reported for bottom-up synthesis of 1D semiconductor nanostructures, whereas synthesized materials are still lacking uniformity in terms of dimensions, morphologies, and doping levels, resulting in inconsistent performance of each device. ${ }^{11-13}$ Furthermore, the techniques currently available for processing as-synthesized 1D nanostructures are unmanageable or incompatible with microfabrication processes. ${ }^{14}$ At present, the traditional thinfilm based processing is quite mature, which has been used successfully in semiconductor technology over the past few decades and led to advancement of modern portable electronics and optoelectronics. Considering the technological compatibility, piezoelectric semiconductor thin films can be a good candidate to substitute $1 \mathrm{D}$ nanostructures for realizing piezotronic applications. There are various methods for growing piezoelectric semiconductor thin films, such as physical vapor deposition, ${ }^{15,16}$ wet chemical deposition, ${ }^{17,18}$ radio frequency (RF) sputtering, ${ }^{19,20}$ molecular beam epitaxy, ${ }^{21,22}$ and metal-organic chemical vapor deposition. ${ }^{23,24}$ Furthermore, wet chemical deposition is a good choice for synthesizing $\mathrm{ZnO}$ thin film with high orientation along the $c$ axis, ${ }^{25}$ which can greatly enhance the piezopotential along the polar axis.

Received: February 17, 2014

Accepted: April 3, 2014 
(a)

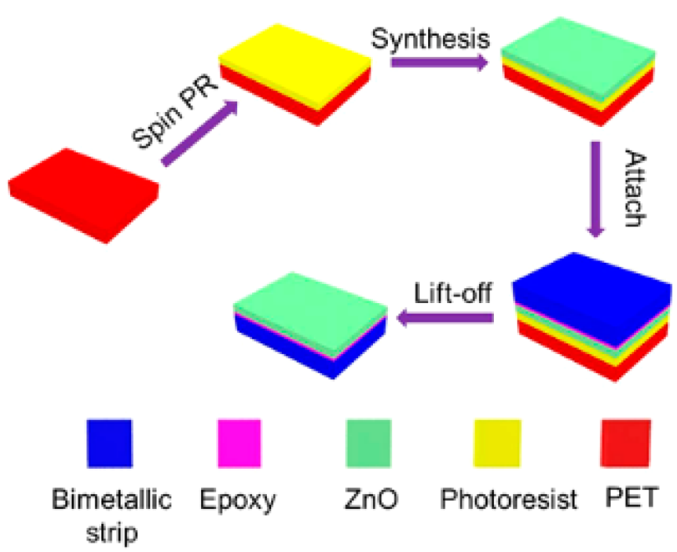

(b)

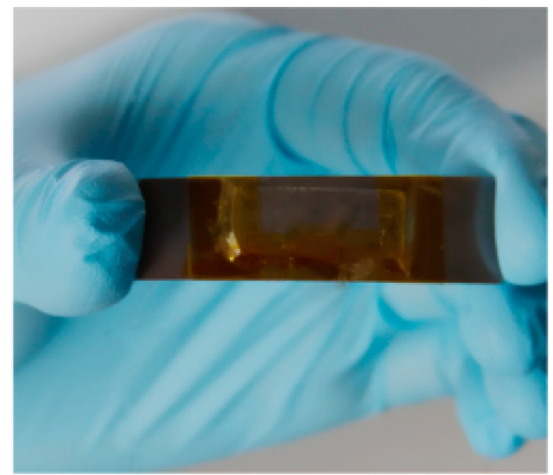

(c)

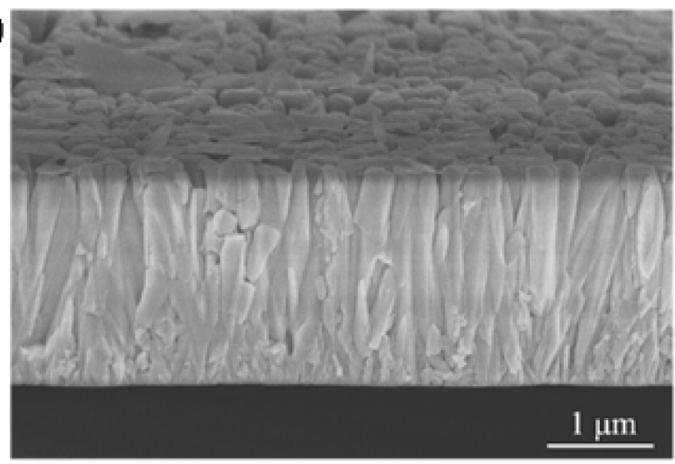

(d)

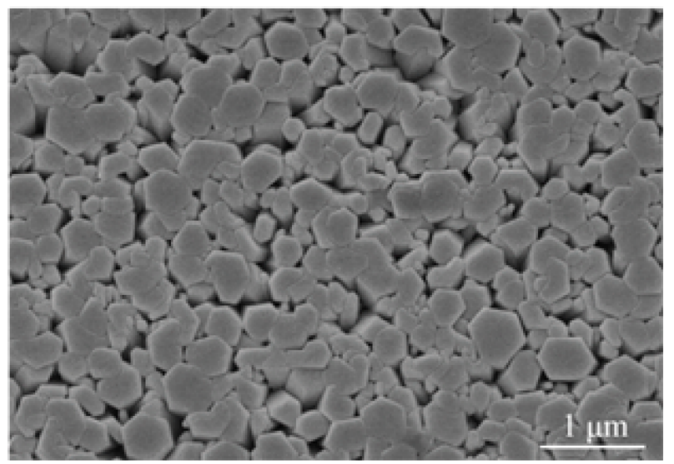

Figure 1. (a) Schematics on how to transfer the ZnO NW film from PET to the bimetallic strip. (b) Photograph of our practical fabricated device. SEM image of the morphology of the as-synthesized $\mathrm{ZnO}$ nanowire film $(\mathrm{c}, \mathrm{d})$.

A measure and control of temperature in an ambient atmosphere is important for our daily life, industrial processes, and environmental monitoring. The bimetallic thermometer is one of the most important candidates for temperature sensing that has outstanding advantages of immunity to electromagnetic interference, simple fabrication, cost-effectiveness, and durability against harsh environments. Piezotronic devices are extremely sensitive to the external applied strain; therefore, by a combination of the piezotronic effect and a bimetallic strip, a temperature sensor with high sensitivity and practicability can be obtained.

In this paper, we present the first study of piezotronic effect as a potential means for measuring temperature by utilizing $\mathrm{ZnO}$ nanowire (NW) film synthesized by the wet chemical deposition method. The developed temperature sensor is characterized under different temperatures in a range from 10 to $110{ }^{\circ} \mathrm{C}$. The thin film is integrated on a temperature sensitive bimetallic strip whose shape changes dramatically once there is a temperature change. The strain produced by the deformation of the strip in responding to temperature change creates piezoelectric polarization charges in the $\mathrm{ZnO} \mathrm{NW}$ film, resulting in a change in Schottky barrier height $(\mathrm{SBH})$ at the metal-semiconductor contact. The piezotronic effect largely enhanced the sensitivity and precision owing to the "gating" effect from the piezoelectric polarization field. A theoretical model using energy band diagrams is proposed to understand the observed behaviors.

\section{EXPERIMENTAL SECTION}

2.1. Synthesis of $\mathrm{ZnO} \mathrm{NW}$ Film. The $\mathrm{ZnO} \mathrm{NW}$ film was synthesized by the wet chemical deposition method on the surface of polyethylene terephthalate (PET) with a thickness of $0.1 \mathrm{~mm}$. Before the growth, the PET was treated with ethanol and deionized water to remove the surface's impurities. Then the as-treated PET coated with a $\mathrm{ZnO}$ seed layer $(20 \mathrm{~nm})$ by RF sputtering was placed in the nutrient solution consisting of zinc nitrate and hexamethylenetetramine (HMTA) with equal concentrations $(0.1 \mathrm{~mol} / \mathrm{L})$. After $4 \mathrm{~h}$ in an oven at $90{ }^{\circ} \mathrm{C}$, the samples were rinsed in deionized water to remove the chemical residuals. Finally the synthesized $\mathrm{ZnO} \mathrm{NW}$ film was transferred to the bimetallic strip, the size of which was $5.5 \mathrm{~cm}$ in length and $2 \mathrm{~cm}$ in width. The selected bimetallic strip was about 120 $\mu \mathrm{m}$ in thickness and had two expansion layers, whose high expansion layer was $\mathrm{Mn}_{75} \mathrm{Ni}_{15} \mathrm{Cu}_{10}$ and low expansion layer was $\mathrm{Ni}_{36} \mathrm{Fe}_{64}$.

2.2. Electrical Measurement of Temperature Sensor. The different temperature environment and the voltage source were provided by a temperature test chamber (Wuxi Zhongtian, GDW-50L) and a synthesized function generator (Stanford Research Systems DS345), respectively. The current signal of the sensor was measured by a low noise current amplifier (Stanford Research Systems SR570).

\section{RESULT AND DISCUSSION}

3.1. Device Fabrication. A transfer technique was introduced to fabricate our device, as schematically shown in Figure 1a. Negative photoresist (SUN-9i) as a sacrificial layer was spin-coated onto the PET substrate before the subsequent synthesis of $\mathrm{ZnO} \mathrm{NW}$ film using the wet chemical deposition method. The bimetallic strip substrate was then attached to the as-synthesized $\mathrm{ZnO} \mathrm{NW}$ film with epoxy. Next, the sample was dried at room temperature for $12 \mathrm{~h}$ to improve epoxy's adhesion to the substrate. Lastly, the sacrificial layer was dissolved by acetone, and the $\mathrm{ZnO} \mathrm{NW}$ film was successfully transferred from the PET substrate to the bimetallic strip substrate. The photograph of the fabricated device was sketched in Figure 1b. This transfer technique overcame the geometric deformation of $\mathrm{ZnO} \mathrm{NW}$ film synthesized directly on the bimetallic strip (Figure S1) for device fabrication. The 

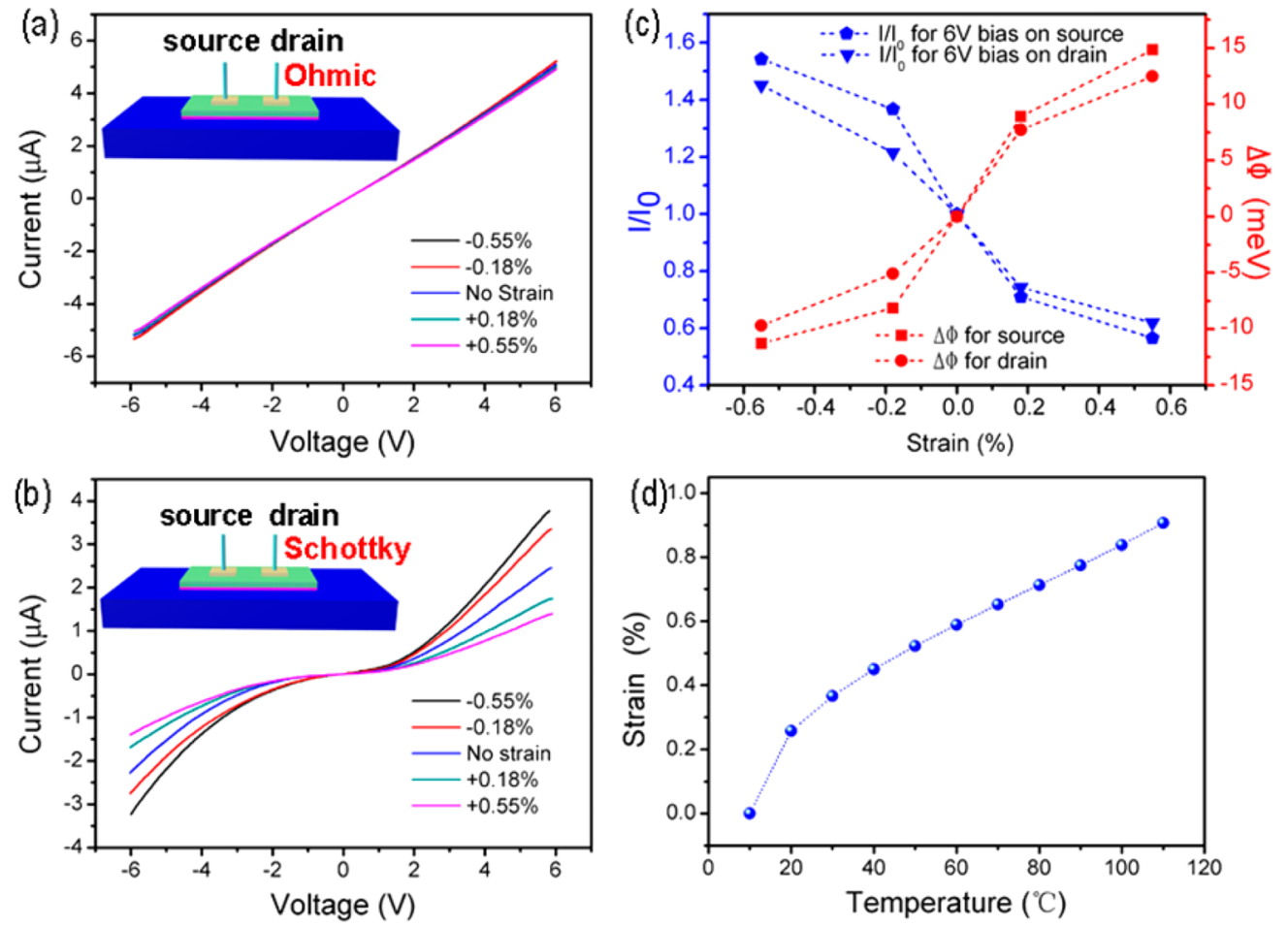

Figure 2. (a) I-V curves of a device with Ohmic contact electrodes under different strain values. (b) I-V curves of a device with Schottky contact electrodes under different strain values. (c) Calculated changes in current and SBHs under different strain values at a fixed bias voltage. (d) Relationship between temperature and strain for the bimetallic strip.

cross-sectional and surface morphologies of the $\mathrm{ZnO} \mathrm{NW}$ film before transfer are presented by scanning electron microscopy (SEM) images in Figures $1 \mathrm{c}$ and d. The nanowires are orderly grown and densely ranged as a textured film with the $c$ axis of the NWs pointing upward. ${ }^{26}$

3.2. Ohmic Contact vs Schottky Contact. There are two effects that can change the transport characteristics of a piezoelectric semiconductor film: piezoresistive effect and piezotronic effect. The piezoresistive effect is a volume effect, and it usually responds linearly to the degree of strain. The piezotronic effect is an interface effect owing to the presence of the piezoelectric polarization charges at the metal-semiconductor interface, which is usually nonlinear. In order to confirm which mechanism plays a primary role in the asfabricated $\mathrm{ZnO} \mathrm{NW}$ film device, a pair of $\mathrm{Al}(30 \mathrm{~nm})$ electrodes was sputtered sequentially onto the first group of $\mathrm{ZnO} \mathrm{NW}$ films on the same sides so as to form Ohmic contacts with $\mathrm{ZnO}$. Considering that there would be high carrier density near the junction interface for Ohmic contact, the piezotronic effect can be significantly attenuated due to the screening effect to the piezoelectric polarization charges, ${ }^{27}$ while geometrical and piezoresistive effects contributing to the conductivity change should remain unaffected. Here, a three-dimensional mechanical manipulation stage with a displacement resolution of $1 \mu \mathrm{m}$ was used to bend the free end of the device to produce a compressive or tensile strain across the thickness direction of the $\mathrm{ZnO} \mathrm{NW}$ film. I-V characteristics were subsequently measured when different strains were exerted on the device, and the results shown in Figure 2a demonstrate that for strain up to $\pm 0.55 \%$, both geometrical and piezoresistive effects have little impact on the device conductivity. The stress states introduced here in the NW film are different from the one stated in the single nanowire piezoelectric test. ${ }^{5,6}$ Since the thickness of the bimetallic strip is much larger than the thickness of the $\mathrm{ZnO}$ film, once the metallic strip is bent inward, the $\mathrm{ZnO}$ film is under tensile strain along the thickness direction (e.g., $c$ axis of the NWs). By the same token, if the bimetallic strip is bent outward, the $\mathrm{ZnO}$ film will have compressive strain along its thickness direction. The magnitude of the strain can be derived using the method introduced previously. ${ }^{13}$

In the second group of devices, silver was used as the metal for the source electrode and the drain electrode to form Schottky contacts, which was confirmed by $\mathrm{I}-\mathrm{V}$ curves as shown in Figure $2 \mathrm{~b}$. The current flowing through the device increased when a compressive strain was applied, while it decreased when a tensile strain was applied. The relative changes in local SBHs were derived by quantifying the $\mathrm{I}-\mathrm{V}$ characteristic curves at a fixed bias voltage V. $I_{0}$ represents the current flowing through the device without strain applied. The $I / I_{0}$ strain curve in Figure $2 \mathrm{c}$ clearly shows that the currents decreased with increasing strain under the reversely biased source or drain (blue hexagons for $6 \mathrm{~V}$ bias on source and blue triangles for $6 \mathrm{~V}$ bias on drain). The thermionic emissiondiffusion theory was utilized to calculate the strain-induced change of $\mathrm{SBH}^{28}{ }^{28}$ which is plotted in Figure $2 \mathrm{c}$ (red roundness and red quadrates). The results show that both SBHs at the source or drain contacts rise when the applied strain increases; a change of $15 \mathrm{meV}$ is received when the applied strain is $0.55 \%$. The bimetallic strip can deform differently with the temperature changing, and the corresponding strain in the film can be directly derived from the shape of the strip. The relationship between temperature and strain is schematically shown in Figure 2d, which indicates that the strain of the bimetallic strip is almost a linear change at a temperature 

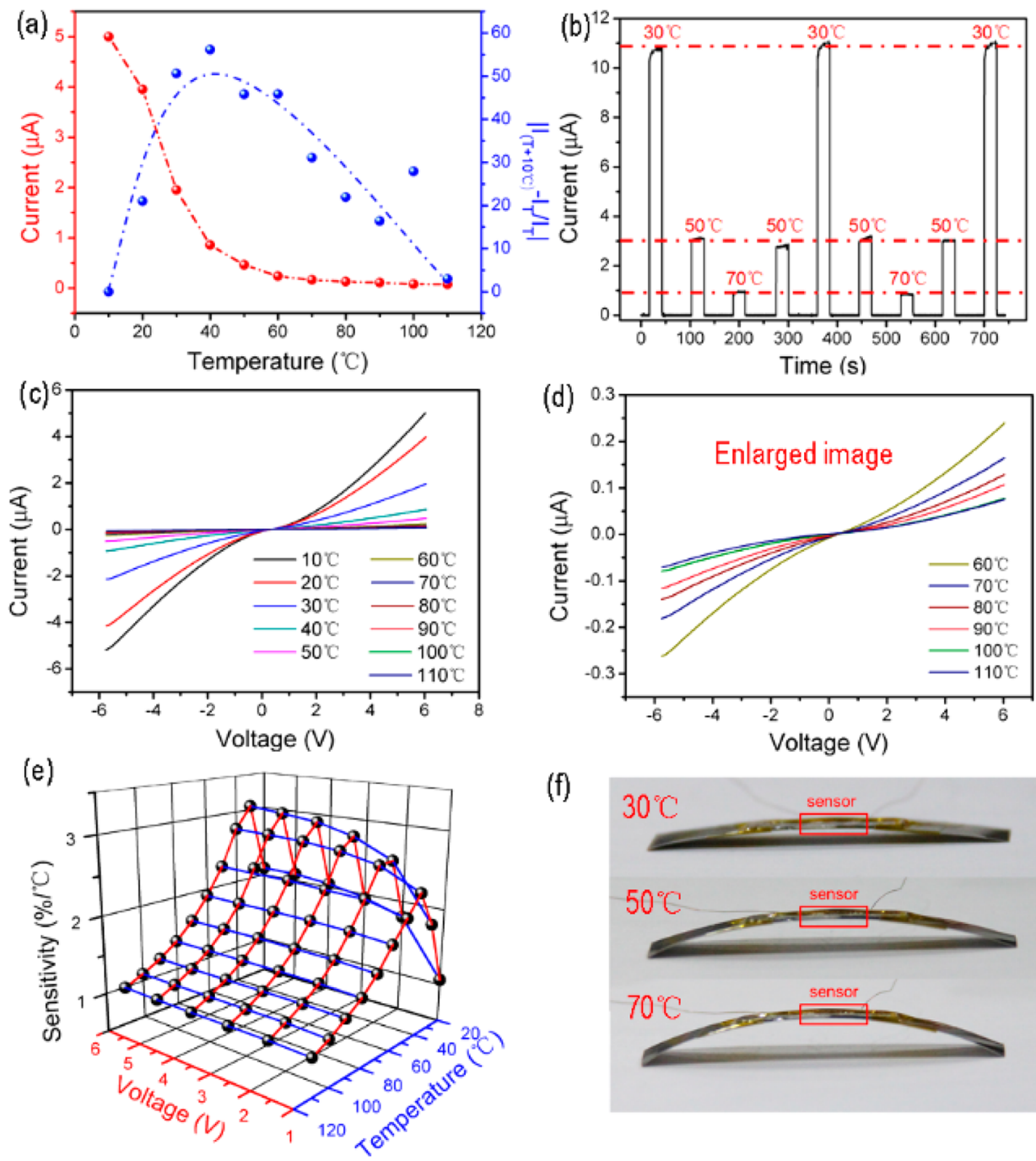

(f)

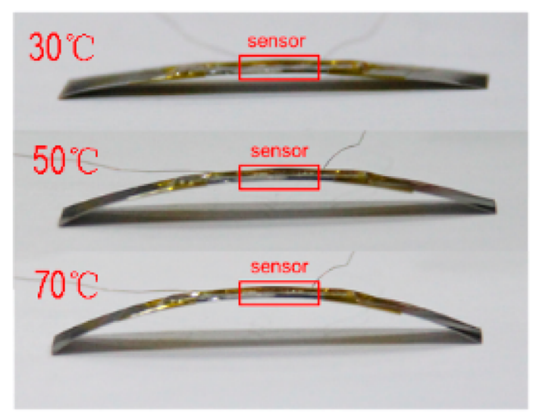

Figure 3. (a) Response and relative current change of the sensor to the temperature, varying from 10 to $110^{\circ} \mathrm{C}$. (b) Repeatability of the temperature sensor at fixed bias voltage. (c) I-V curves of the sensor at different temperatures. (d) Enlarged image of c from 60 to $110{ }^{\circ} \mathrm{C}$. (e) The normalized average sensitivity of the sensor at different bias voltages and different temperatures, which was defined as follows: $S=I\left(I_{\text {temperature }}-I_{10^{\circ} \mathrm{C}}\right) /\left(I_{10^{\circ} \mathrm{C}} \times\right.$ $\Delta T) \mathrm{I}, \Delta T=$ temperature $-10^{\circ} \mathrm{C}$. (f) Deformation of the practical sensor at different temperatures. The bimetallic strip was $5.5 \mathrm{~cm}$ in length and 2 $\mathrm{cm}$ in width, while the $\mathrm{ZnO} \mathrm{NW}$ film was about $1 \mathrm{~cm}$ in length and $0.5 \mathrm{~cm}$ in width.

between 20 and $110^{\circ} \mathrm{C}$ and could exert effective strain on the $\mathrm{ZnO} \mathrm{NW}$ film.

\subsection{Characterization of the Sensor at Different}

Temperatures. The response of the $\mathrm{ZnO} \mathrm{NW}$ film based sensor to the temperature, measured at a fixed bias of $6 \mathrm{~V}$, is presented in Figure 3a (red roundness). The response signal of the sensor (i.e., current here) decreased significantly from a few microamperes to dozens of nanoamperes with the increase of the temperature from 10 to $110{ }^{\circ} \mathrm{C}$. This obvious trend is mainly controlled by the piezoelectric polarization charges at the contact interface and thermal disturbance of the sensor. The induced negative piezoelectric polarization charges at the contact interface between silver and $\mathrm{ZnO} \mathrm{NW}$ film raise the $\mathrm{SBH}$ and reduce the current coupled with thermal disturbance. This result indicates that the $\mathrm{ZnO} \mathrm{NW}$ film based device has a good response to temperature. The relative current change of this sensor was calculated utilizing eq 1, as shown in Figure 3a (blue roundness), which presents a trend of parabola, and the relative current change is as high as $50 \%$ from 30 to $70{ }^{\circ} \mathrm{C}$.

$$
\Delta I / I=\left(I_{\text {temperature }}-I_{\text {temperature }-10^{\circ} \mathrm{C}}\right) / I_{\text {temperature }-10^{\circ} \mathrm{C}}
$$

where $I_{\text {temperature }}$ is the current signal value at a temperature and $I_{\text {temperature }-10^{\circ} \mathrm{C}}$ at a temperature which is $10^{\circ}$ lower. The $\mathrm{ZnO}$ NW film based sensor for the temperature change showed good repeatability with a fixed bias voltage at the source electrode, as illustrated in Figure 3b. This temperature sensor could keep a stable value at a certain temperature in the cycle of the temperature change.

Systematic measurements of the temperature sensor were performed in a temperature test chamber under different temperatures at a fixed relative humidity about $12 \%$. Figure $3 c$ shows the temperature sensor's I-V characteristic at different temperatures. The device presents a typical nonlinear symmetrical I-V curve, which indicates that the two Schottky barriers at the two contacts have almost the same heights under the fixed bias voltage. To further demonstrate the $\mathrm{ZnO} N W$ film based temperature sensor's performance, the sensitivity under different source bias voltages and different temperatures derived from Figure $3 \mathrm{c}$ was plotted in a $3 \mathrm{D}$ graph, as shown in 

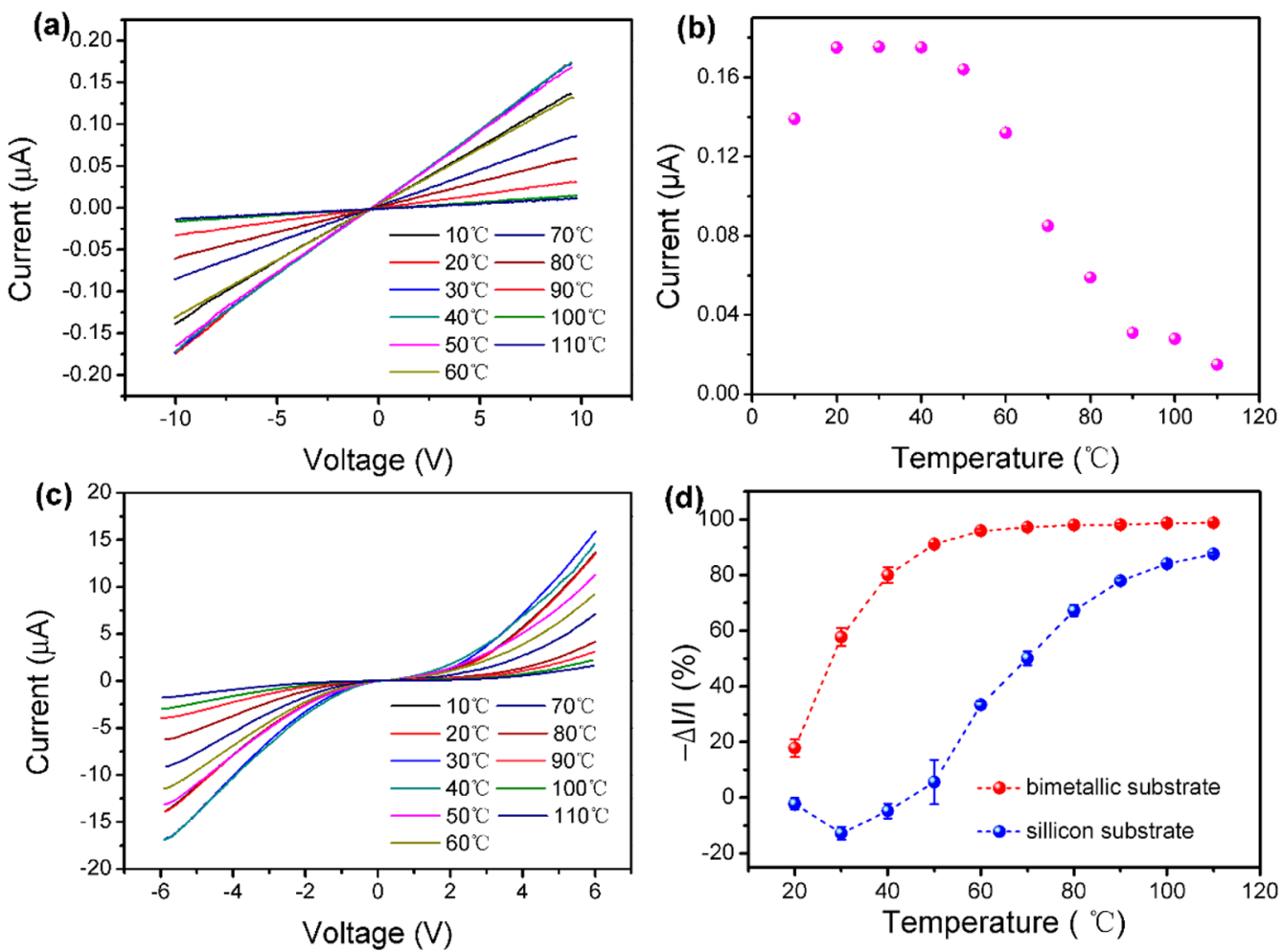

Figure 4. (a) I-V curves of the device with Ohmic contact at different temperatures. (b) The current signals of the device with Ohimc contact under a fixed bias voltage at different temperatures. (c) I-V curves of the device with Schottky contact and sillicon substrate at different temperatures. (d) Normalized current change at different temperatures. The blue curve represents the device with a silicon substrate; the red represents the device with a bimetallic strip substrate obtained from Figure 3c.

Figure 3e. Furthermore, a more detailed characterization about the changing trends of the sensitivity and the resolution are illustrated in Figures S2 and S3, respectively. On the whole, at one fixed temperature, the sensitivity gradually increased with the increase of bias voltages, among which the maximum one and the minimum one incredibly reached $3.05 \% /{ }^{\circ} \mathrm{C}$ and $0.98 \% /{ }^{\circ} \mathrm{C}$, which were about 10 times and 3 times, respectively, higher than responses of the temperature sensors on the market, such as PT100 based thermometers. At one fixed bias voltage, the sensitivity first increased to the maximum value with the rise of temperature and then gradually decreased when the temperature exceeded $30{ }^{\circ} \mathrm{C}$, which is determined by the properties of the bimetallic strip. The deformations of our practical device under different temperatures are shown in Figure 3f.

3.4. Piezotronic Enhanced Temperature Sensor. In order to research whether the current change of the temperature sensor was affected by the change of $\mathrm{SBH}$, the mobility of carriers or the piezopotential, two group contrast devices were fabricated. On one hand, a device that sputtered a $30 \mathrm{~nm}$ aluminum electrode to form Ohmic contact was characterized under different bias voltages and different temperatures as shown in Figure 4a. The variation of the current signal under a fixed bias voltage is illustrated in Figure $4 \mathrm{~b}$, fitting a parabolic function with the temperature, which indicates that, with the increase of the temperature, the mobility of the carriers (electron) increases at low temperatures and then decreases at high temperatures and practically accords with the previous research. ${ }^{29}$ On the other hand, the $\mathrm{ZnO} \mathrm{NW}$ films were transferred to silicon and aluminum oxide to fabricate contrast devices with Schottky contact, both of which had a low coefficient of thermal expansion. All of the devices exhibited similar current changes under different temperatures. Here, taking a silicon substrate based device as an example, the $\mathrm{I}-\mathrm{V}$ curves are illustrated in Figure 4c. The possible explanation for the experimental results is as follows: At low temperatures, with the increase of temperature, the Fermi level and band gap of the metal and semiconductor remain little changed while the mobility of the carrier (electron) increases rapidly, ${ }^{29}$ resulting in the increased current. At high temperatures, with the increase of temperature, the mobility of the carrier decreases gradually while the lowered Fermi level and the shrinkage band gap lead to the rise of the Schottky barriers, resulting in the decreased current.

Further detailed current change compared with the bimetallic strip substrate based temperature sensor was defined as eq 2 and illustrated in Figure 4d. The bimetallic strip based device exhibits a more distinct current change than the silicon based one, which is enhanced by piezotronic effect, which improves the performance of this temperature sensor obviously.

$$
-\Delta I / I=\left(I_{10^{\circ} \mathrm{C}}-I_{\text {temperature }}\right) / I_{10^{\circ} \mathrm{C}}
$$

3.5. Theoretical Basis. The band diagrams of the $\mathrm{ZnO} N W$ film based temperature sensor are illustrated in Figure 5 to better understand the underlying working mechanism. Figure 5a shows the original state of the energy band with two similar SBHs formed at both contacts. When the sensor is connected to an external power source, the quasi-Fermi level of the negative connected electrode (source side here) is raised, giving rise to the higher potential drop, as shown in Figure 5b. However, the biased voltage does not affect both of the barrier heights between the electrode and the $\mathrm{ZnO} \mathrm{NW}$ film. The bimetallic strip deforms correspondingly to the temperature 
(a)
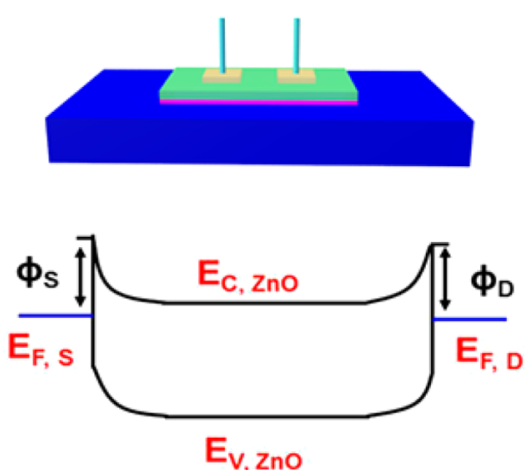

(c)
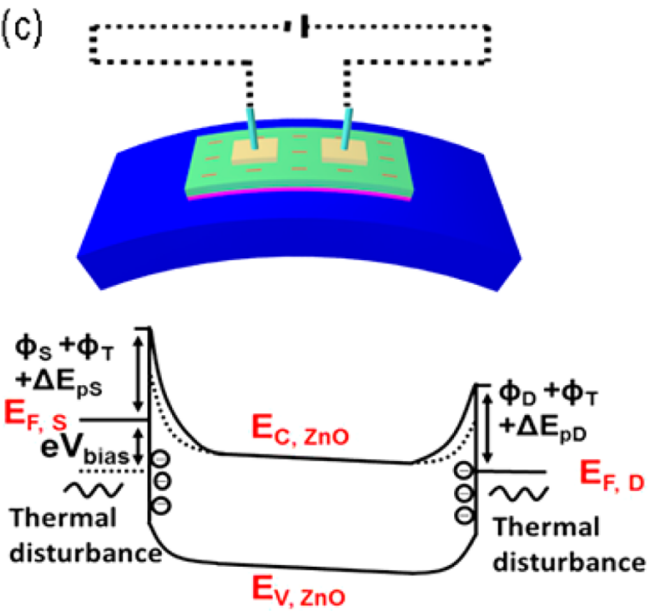

(b)
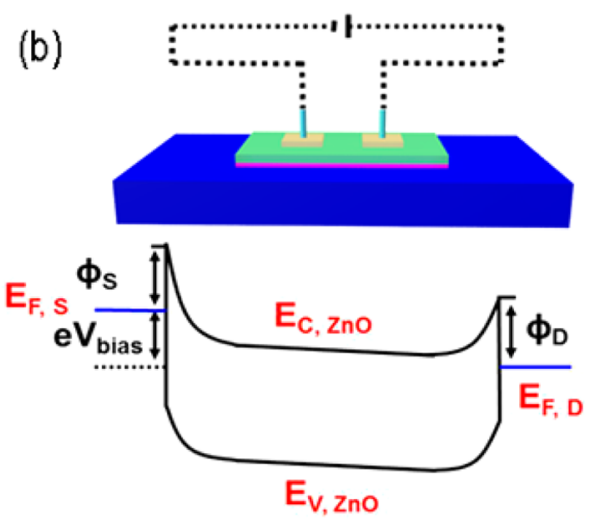

(d)

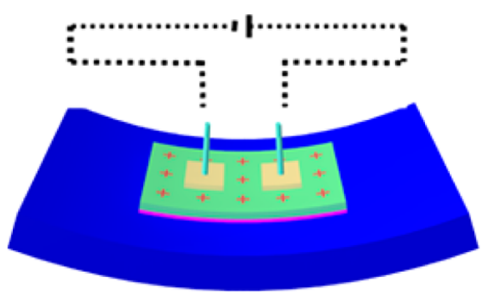

$\phi_{\mathrm{S}}-\phi_{\mathrm{T}}$

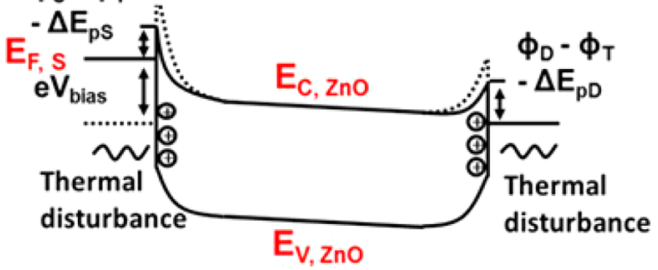

Figure 5. Schematic energy band diagrams illustrating the change of Schottky barriers at the source and drain contacts. (a) Similar Schottky barrier heights were formed at both contacts. (b) The quasi-Fermi level of one electrode is raised (source side here) with an external power source applied. (c) When the temperature is raised, negative piezoelectric polarization charges are induced at the top surface of the $\mathrm{ZnO}$ film, increasing the SBHs coupled with thermal disturbance at both contacts. (d) When the temperature is lowered, positive piezoelectric polarization charges are induced at the top surface of the $\mathrm{ZnO}$ film, decreasing the SBHs coupled with thermal disturbance at both contacts.

rise, which is equivalent to applying a tensile strain in the thickness direction of the $\mathrm{ZnO} \mathrm{NW}$ film; therefore, negative piezoelectric polarization charges are induced at the top surface of $\mathrm{ZnO} \mathrm{NW}$ film if its normal direction is the $c$ axis. These immobile ionic charges deplete part of the free electrons near the metal-semiconductor interface and therefore raise the SBH coupled with the thermal disturbance at both contacts, as shown in Figure 5c. When the temperature was lowered, the bimetallic strip posed tensile strain in the thickness direction. Accordingly, positive piezoelectric polarization charges are induced near the top surface of the $\mathrm{ZnO} \mathrm{NW}$ film, attracting free electrons toward the metal-semiconductor interface and subsequently decreasing the SBHs coupled with the thermal disturbance at both contacts, as shown in Figure 5d. The conductivity of the entire NW film device hinges on the reversely biased Schottky barrier and mobility of the carrier. Additionally, effective conductivity of the device is sensitive to the change in SBH at that contact. Different from piezotronic devices based on 1D nanomaterials in which metal-semiconductor contacts are formed at the two opposite polar surfaces, ${ }^{5,6}$ both electrode contacts are at the same surface of asgrown $\mathrm{ZnO} \mathrm{NW}$ film. Thus, piezoelectric polarization charges with the same polarity will be induced at the two Schottky contacts when external strain is applied on the $\mathrm{ZnO}$ NW film. This led to the observed $\mathrm{I}-\mathrm{V}$ curves (Figures $3 \mathrm{c}$ and $\mathrm{d}$ ) in which there is the same tuning trend of applied strain when either source or drain side is reversely biased.

\section{CONCLUSION}

In summary, via a film transfer technique, we demonstrated the first flexible piezotronic temperature sensor, which was based on wet chemical grown $\mathrm{ZnO}$ piezoelectric $\mathrm{NW}$ film. The primary role of the piezotronic effect over the geometrical and piezoresistive effects in the as-fabricated devices has been confirmed, and the piezotronic effect on charge carrier transport under different strains is subsequently studied. In addition, we also showed that the temperature sensing capability of an as-fabricated nanowire film based piezotronic device can be tuned by piezopotential, exhibiting dramatically enhanced sensitivity. A theoretical model is proposed to interpret the observed behaviors of the sensor. This study provides an effective means to fabricate a temperature sensor with higher performance based on the piezotronic effect in the future.

\section{ASSOCIATED CONTENT}

\section{S Supporting Information}

Additional schematic figures on the $\mathrm{I}-\mathrm{V}$ curves of the sensor where $\mathrm{ZnO} \mathrm{NW}$ film was directly synthesized on the bimetallic strip, the sensitivity, and the resolution of the temperature 
sensor. This material is available free of charge via the Internet at http://pubs.acs.org.

\section{AUTHOR INFORMATION}

\section{Corresponding Author}

*E-mail: zlwang@gatech.edu.

\section{Author Contributions}

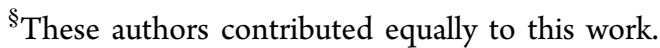

\section{Notes}

The authors declare no competing financial interest.

\section{ACKNOWLEDGMENTS}

This research was supported by the "thousands talents" program for the pioneer researcher and his innovation team, China, and Beijing City Committee of science and technology projects (Z131100006013004, Z131100006013005).

\section{REFERENCES}

(1) Wang, Z. L.; Song, J. Piezoelectric Nanogenerators Based on Zinc Oxide Nanowire Arrays. Science 2006, 312, 242-246.

(2) Espinosa, H. D.; Bernal, R. A.; Minary-Jolandan, M. A Review of Mechanical and Electromechanical Properties of Piezoelectric Nanowires. Adv. Mater. 2012, 24, 4656-4675.

(3) Su, W. S.; Chen, Y. F.; Hsiao, C. L.; Tu, L. W. Generation of Electricity in GaN Nanorods Induced by Piezoelectric Effect. Appl. Phys. Lett. 2007, 90, 063110.

(4) Wang, Z. L. Nanopiezotronics. Adv. Mater. 2007, 19, 889-892.

(5) Zhou, J.; Gu, Y. D.; Fei, P.; Mai, W. J.; Gao, Y. F.; Yang, R. S.; Bao, G.; Wang, Z. L. Flexible Piezotronic Strain Sensor. Nano Lett. 2008, 8, 3035-3040.

(6) Wang, X. D.; Zhou, J.; Song, J. H.; Liu, J.; Xu, N. S.; Wang, Z. L. Piezoelectric Field Effect Transistor and Nanoforce Sensor Based on a Single ZnO Nanowire. Nano Lett. 2006, 6, 2768-2772.

(7) Wu, W.; Wen, X.; Wang, Z. L. Taxel-addressable Matrix of Vertical-nanowire Piezotronic Transistors for Active and Adaptive Tactile Imaging. Science 2013, 340, 952-957.

(8) Pan, C. F.; Yu, R. M.; Niu, S. M.; Zhu, G.; Wang, Z. L. Piezotronic Effect on the Sensitivity and Signal Level of Schottky Contacted Proactive Micro/Nanowire Nanosensors. ACS Nano 2013, 7, 1803-1810.

(9) Niu, S.; Hu, Y.; Wen, X.; Zhou, Y.; Zhang, F.; Lin, L.; Wang, S.; Wang, Z. L. Enhanced Performance of Flexible ZnO Nanowire Based Room-temperature Oxygen Sensors by Piezotronic Effect. Adv. Mater. 2013, 25, 3701-3706.

(10) Yu, R.; Pan, C.; Wang, Z. L. High Performance of $\mathrm{ZnO}$ Nanowire Protein Sensors Enhanced by the Piezotronic Effect. Energy Environ. Sci. 2013, 6, 494-499.

(11) Allen, J. E.; Perea, D. E.; Hemesath, E. R.; Lauhon, L. J. Nonuniform Nanowire Doping Profiles Revealed by Quantitative Scanning Photocurrent Microscopy. Adv. Mater. 2009, 21, 3067-3072.

(12) Perea, D. E.; Hemesath, E. R.; Schwalbach, E. J.; Lensch-Falk, J. L.; Voorhees, P. W.; Lauhon, L. J. Direct Measurement of Dopant Distribution in an Individual Vapor-liquid-solid Nanowire. Nat. Nanotechnol. 2009, 4, 315-319.

(13) Wen, X.; Wu, W.; Ding, Y.; Wang, Z. L. Piezotronic Effect in Flexible Thin-film Based Devices. Adv. Mater. 2013, 25, 3371-3379.

(14) Freer, E. M.; Grachev, O.; Duan, X. F.; Martin, S.; Stumbo, D. P. High-yield Self-limiting Single-nanowire Assembly with Dielectrophoresis. Nat. Nanotechnol. 2010, 5, 525-530.

(15) McMurran, J.; Kouvetakis, J.; Smith, D. J. Development of a Low-temperature GaN Chemical Vapor Deposition Process Based on a Single Molecular Source $\mathrm{H}_{2} \mathrm{GaN}_{3}$. Appl. Phys. Lett. 1999, 74, 883885.

(16) Lu, J. G.; Kawaharamura, T.; Nishinaka, H.; Kamada, Y.; Ohshima, T.; Fujita, S. ZnO-based Thin Films Synthesized by
Atmospheric Pressure Mist Chemical Vapor Deposition. J. Cryst. Growth 2007, 299, 1-10.

(17) Wei, Y.; Wu, W.; Guo, R.; Yuan, D.; Das, S.; Wang, Z. L. Waferscale High-throughput Ordered Growth of Vertically Aligned $\mathrm{ZnO}$ Nanowire Arrays. Nano Lett. 2010, 10, 3414-3419.

(18) Ilican, S.; Caglar, Y.; Caglar, M. Preparation and Characterization of $\mathrm{ZnO}$ Thin Films Deposited by Sol-gel Spin Coating Method. J. Optoelectron. Adv. Mater. 2008, 10, 2578-2583.

(19) Tominaga, K.; Umezu, N.; Mori, I.; Ushiro, T.; Moriga, T.; Nakabayashi, I. Transparent Conductive $\mathrm{ZnO}$ Film Preparation by Alternating Sputtering of $\mathrm{ZnO}: \mathrm{Al}$ and $\mathrm{Zn}$ or $\mathrm{Al}$ Targets. Thin Solid Films 1998, 334, 35-39.

(20) Carcia, P. F.; McLean, R. S.; Reilly, M. H.; Nunes, G. Transparent ZnO Thin-film Transistor Fabricated by RF Magnetron Sputtering. Appl. Phys. Lett. 2003, 82, 1117-1119.

(21) Chen, Y. F.; Bagnall, D. M.; Zhu, Z. Q.; Sekiuchi, T.; Park, K. T.; Hiraga, K.; Yao, T.; Koyama, S.; Shen, M. Y.; Goto, T. Growth of ZnO Single Crystal Thin Films on c-plane (ll $\left.\begin{array}{llll}0 & 0 & 0 & 1\end{array}\right)$ Sapphire by Plasma Enhanced Molecular Beam Epitaxy. J. Cryst. Growth 1997, 181, 165169.

(22) Hughes, W. C.; Rowland, W. H.; Johnson, M. A. L.; Fujita, S.; Cook, J. W.; Schetzina, J. F.; Ren, J.; Edmond, J. A. Molecular-Beam Epitaxy Growth and Properties of GaN Films on GaN/SiC Substrates. J. Vac. Sci. Technol. B 1995, 13, 1571-1577.

(23) Emanetoglu, N. W.; Gorla, C.; Liu, Y.; Liang, S.; Lu, Y. Epitaxial $\mathrm{ZnO}$ Piezoelectric Thin Films for Saw Filters. Mater. Sci. Semicond. Process. 1999, 2, 247-252.

(24) Nakamura, S.; Harada, Y.; Seno, M. Novel Metalorganic Chemical Vapor Deposition System for GaN Growth. Appl. Phys. Lett. 1991, 58, 2021-2023.

(25) Chen, L.-Y.; Yin, Y.-T.; Chen, C.-H.; Chiou, J.-W. Influence of Polyethyleneimine and Ammonium on the Growth of $\mathrm{ZnO}$ Nanowires by Hydrothermal Method. J. Phys. Chem. C 2011, 115, 20913-20919.

(26) Vayssieres, L. Growth of Arrayed Nanorods and Nanowires of $\mathrm{ZnO}$ from Aqueous Solutions. Adv. Mater. 2003, 15, 464-466.

(27) Gao, Y.; Wang, Z. L. Equilibrium Potential of Free Charge Carriers in a Bent Piezoelectric Semiconductive Nanowire. Nano Lett. 2009, 9, 1103-1110.

(28) Zhang, Y.; Liu, Y.; Wang, Z. L. Fundamental Theory of Piezotronics. Adv. Mater. 2011, 23, 3004-3013.

(29) Size, S. M.; Ng, K. K. Physics of Semiconductor Devices; John Wiley \& Sons: Hoboken, NJ, 1981. 